\title{
A prediction rule identified patients with atrial fibrillation at low risk of stroke while taking aspirin
}

van Walraven C, Hart RG, Wells GA, et al. A clinical prediction rule to identify patients with atrial fibrillation and a low risk for stroke while taking aspirin. Arch Intern Med 2003;163:936-43.

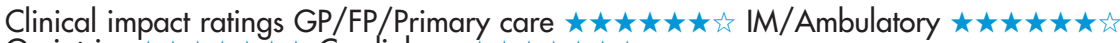

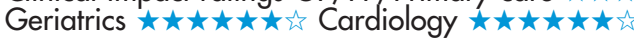

What is the accuracy of an age independent clinical prediction rule for identifying patients with non-valvular atrial fibrillation (AF) who are at low risk of all cause stroke or transient ischaemic attack (TIA) while taking aspirin?

\section{METHODS}

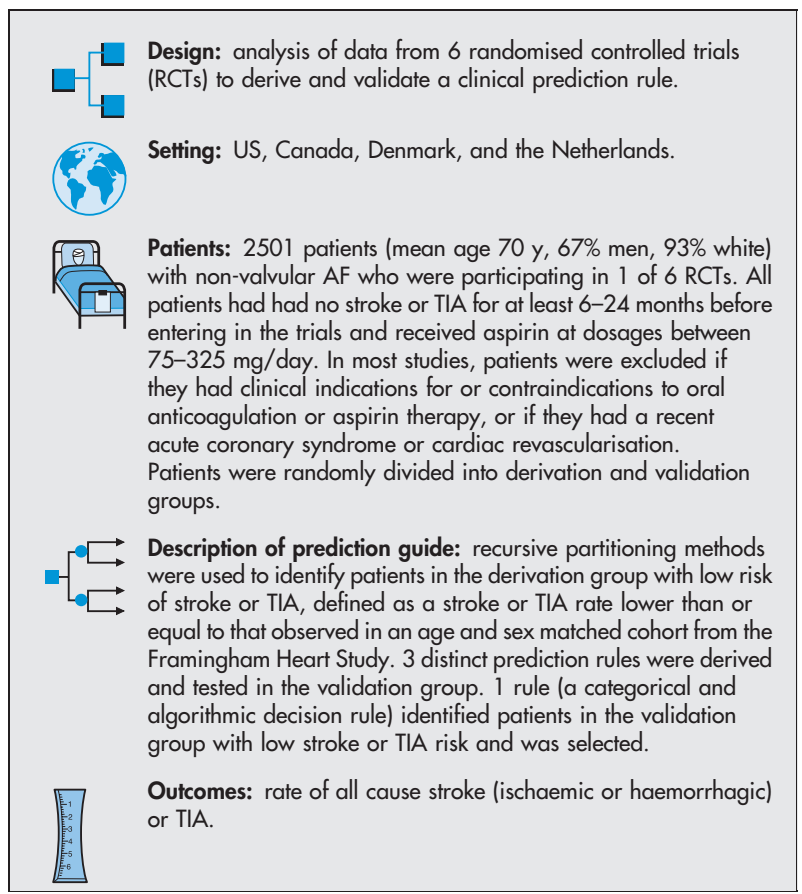

\section{MAIN RESULTS}

Overall, 166 patients $(6.6 \%)$ had a stroke or TIA during 4689 person years (PYs) of observation for an incident rate of 3.5 events per 100 PYs. In the derivation group, 4 factors identified patients with low risk of stroke or TIA: (1) no previous stroke or TIA, (2) no treated hypertension or systolic blood pressure $\geqslant 140 \mathrm{~mm} \mathrm{Hg}$, (3) no previous myocardial infarction or angina, and (4) no diabetes. In the derivation group, patients classified as having low risk of stroke or TIA had 1.0 events per 100 PYs, compared with an age and sex matched rate of 1.2 events per 100 PYs. In the validation group, patients classified as having low risk of stroke or TIA had 1.1 events per 100 PYs, compared with an age and sex matched rate of 1.2 events per 100 PYs. Overall, the prediction rule classified 588 (23.5\%) patients as having low risk of stroke or TIA, of which $144(24.5 \%)$ were $>75$ years of age.

\section{CONCLUSION}

An age independent clinical prediction rule identified patients with non-valvular atrial fibrillation at low risk of all cause stroke or transient ischaemic attack while taking aspirin.

Abstract and commentary also appear in ACP Journal Club.

\section{Commentary}

Cor every 100 patients in the community with non-valvular AF, about 5 will have a stroke each year. ${ }^{1}$ This is about 5 times greater than people of the same age and sex in the community who do not have AF; their annual stroke rate is about $1 \%{ }^{2}$ Treating all 100 patients with AF with oral anticoagulation would reduce the stroke rate by two thirds to $2 \%$, thus saving 3 strokes per year, but at the expense of causing 1 or 2 serious bleeds. Treating all 100 patients with AF with aspirin would reduce the stroke rate by one fifth to $4 \%$, thus saving 1 stroke per year, but with less risk of bleeding

In an ideal world, we would be able to identify the $5 \%$ of patients with AF who will have a stroke each year and treat them with the more effective but more risky oral anticoagulation, and treat the remainder with low risk treatments such as aspirin, or no treatment. Such predictions, however, are difficult to make in individual patients.

van Walraven et al have developed a clinical prediction model that reliably identifies patients with $\mathrm{AF}$ who, when taking aspirin, will have an acceptably low risk of stroke (a risk that is less than or equal to persons of the same age and sex in the community without AF).

For clinicians, the beauty of this model is that it is simple and can be generated in the office or at the bedside. It requires that patients have none of the following 4 clinical features: symptomatic cerebrovascular disease (previous stroke or TIA), symptomatic coronary heart disease, diabetes, or treated hypertension or systolic blood pressure $>140 \mathrm{~mm}$ Hg. Patients can therefore be identified after 4 questions plus or minus a measurement of blood pressure and blood glucose concentration. Furthermore, the model is independent of age, internally valid, and robust.

Graeme J Hankey, MBBS, MD, FRCP Royal Perth Hospital and University of Western Australia Perth, Western Australia, Australia

1 Flegel KM, Shipley MJ, Rose G. Risk of stroke in non-rheumatic atrial fibrillation. Lancet 1987;1:526-9.

2 Wolf PA, Abbott RD, Kannel WB. Atrial fibrillation as an independent risk factor for stroke: the Framingham Study. Stroke 1991;22:983-8. 